\title{
Solid-phase luminescence of pyrene in chitosan adsorbents
}

\author{
Danila A. Tatarinov*, Sofia R. Sokolnikova, and Natalia A. Myslitskaya \\ Department of Physics, Kaliningrad State Technical University, 1 Sovetsky prospect, Kaliningrad 236022, Russia \\ * e-mail: dan.tatarinov@mail.ru
}

\begin{abstract}
The preparation method of modified chitosan films for adsorption of pyrene from solution is described. Solid-phase luminescence of samples was studied, degree of extraction and index of polarity of microenvironment of pyrene molecules was determined. It has been proven possible to carry out luminescent analysis of substances for pyrene content using made films. The observed results demonstrate an ability for the using method of solid-phase luminescence (SPL) to increase the range of optical transparency and avoid pre-extraction and concentration of the test samples. (C) 2020 Journal of Biomedical Photonics \& Engineering.
\end{abstract}

Keywords: Luminescent analysis; pyrene; polycyclic aromatic hydrocarbons; surfactants; solid-phase luminescence; chitosan.

Paper \#3342 received 12 Jan 2020; revised manuscript received 19 Mar 2020; accepted for publication 19 Mar 2020; published online 25 Mar 2020. doi: 10.18287/JBPE20.06.010305.

\section{Introduction}

Nowadays, polycyclic aromatic hydrocarbons (PAH) are one of the most dangerous carcinogens. The reason for PAH formation is pyrolysis of organic substances. The mechanism of toxicity is considered to be interference with the function of cellular membranes as well as with enzyme systems that are associated with the membrane. It has been proved that PAH can cause carcinogenic and mutagenic effects and are potent immunosuppressants. Effects have been documented on immune system development, humoral immunity and on host resistance. PAH are accumulated in the human body and cause a change in the structure of DNA, which results in cancer. [1]. In this regard, the selected topic is relevant in modern environmental conditions for the protection of human health [2-4].

Due to their bioaccumulation capacity, substances of the PAH group are potentially dangerous for humans even at low concentrations. According to Russian standards for monitoring PAH content, the level of content of one of the most common representatives of these substances is measured - benz(a)pyrene (BP) [5]. Maximum permissible average daily concentrations of $\mathrm{BP}$ in atmospheric air is $0.001 \mathrm{mg} / \mathrm{l}$, in soil is $0.02 \mathrm{mg} / \mathrm{kg}$, for majority of food is not more than $1 \mu \mathrm{g} / \mathrm{kg}[6]$.

In laboratory conditions the representative of PAH pyrene was the most widely used for the study of PAH content in environmental objects. It has features of the electron-vibrational structure of its radiation spectrum, which is characterized by five well resolved main vibrational bands [2]. Also, pyrene has similar fluorescence spectra to one of the most dangerous $\mathrm{PAH}-\mathrm{BP}$ but is not so toxic that it facilitates its use in laboratory studies [3].

Determination of PAH content is a complex analytical task due to low concentrations of these substances in the environment, and modern methods of analysis are used to solve it [7]. High-performance liquid chromatography is most commonly used for $\mathrm{PAH}$ detection. Significant disadvantages of this method are the high cost and the time required to analyze samples. Recent scientific developments make it possible to create more sensitive, reliable and express methods of $\mathrm{PAH}$ control. One developing method of PAH detection is luminescent analysis [8].

It has been previously found [7,9] that in order to increase luminescence intensity, it is effective to use a micellar concentration of pyrene in aqueous solution, but this method allows us to study only liquid substances having sufficient optical transparency. Therefore, SPL was chosen to study substances with large optical transparency ranges.

The SPL method is widely used to determine many inorganic and organic compounds [10-11]. SPL allows us to combine sorption concentration of substances (solid-phase extraction) with their next luminescent determination directly in the sorbent phase that increases sensitivity of the method. Thus, it is possible to research samples in different aggregate states and having different optical transparency [2].

The aim of the study is to research the luminescent properties of pyrene in a fluorescent assay method using 
solid chitosan matrices and to study the question of their further modification.

\section{Materials and methods}

Various materials are used as bases for solid matrices, such as cellulose [12], cellulose acetates [13], chitosan [14]. One of the most promising bases is chitosan, it is a polysaccharide produced by partial deacetylation of chitin, which is a structural element of the exoskeleton of insects, crustaceans and cell walls of fungi [15]. Chitosan is producing in large quantities, thus it has a low cost. It is known chitosan matrices exhibit high sorption capacity [16] and are effective sorbents of heavy metal ions, surfactants, PAHs, etc. [14].

In this study chitosan (Diaham LLC, 95\% deacetylation degree) was used as a base for a solid matrix. Chitosan solution at a concentration of $25 \mathrm{~g} / 1$ was prepared in a $2 \%$ acetic acid solution. The solution were poured into each Petri dish and allowed to dry at room temperature for $72 \mathrm{~h}$ (Fig. 1a). Also, a few samples were modified with polyvinyl alcohol (PVA) and the surfactant cetyltrimethylammonium bromide (CTAB).

After drying chitosan-based films were kept in 5\% aqueous $\mathrm{KOH}$ solution to convert the biopolymer to the basic (hydrophobic) form (Fig. 1b). The samples were washed in distilled water with $\mathrm{pH}=7$ and dried at a temperature of $\mathrm{t}=20^{\circ} \mathrm{C}$ and a relative humidity of $60 \%$ for further studies.

Dimensions of films after drying are $\mathrm{d}=5 \mathrm{~cm}$, thickness $\mathrm{h}=0.1 \div 0.3 \mathrm{~mm}$. After the films are made hydrophobic, the linear dimensions increase slightly. For luminescent analysis $1 \times 1 \mathrm{~cm}$ samples were prepared from the films and fixed to the slide.

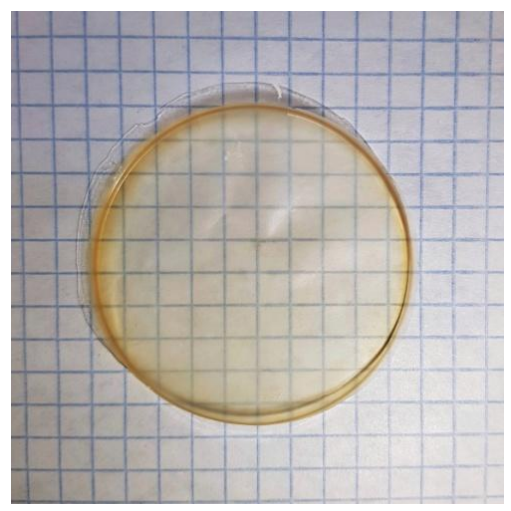

Fig. 1 Chitosan film in hydrophobic form.

A modular system HORIBA Fluorolog-3 TCSPC was used for luminescent analysis. The modular system is shown in Fig. 2. Error of measured parameters consists of error of emission monochromator and error of luminescence intensity measurement. The HORIBA manufacturer stated error value of monochromator emission $\pm 0.5 \mathrm{~nm}[17]$.

Statistical processing of experimental results was performed using the method IUPAC [18]. For a sample population with degrees of freedom $n=5$, root mean square deviation was calculated. Then confidence interval about the mean with confidence level 0.95 and error of result were determined. For the polarity index the absolute error was $0.03-0.05$, and for the extent of pyrene extraction the absolute error was $2-4 \%$.

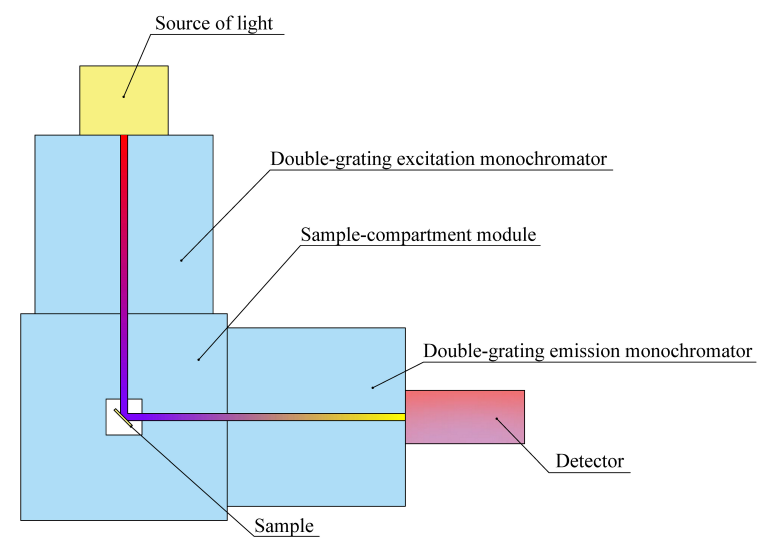

a

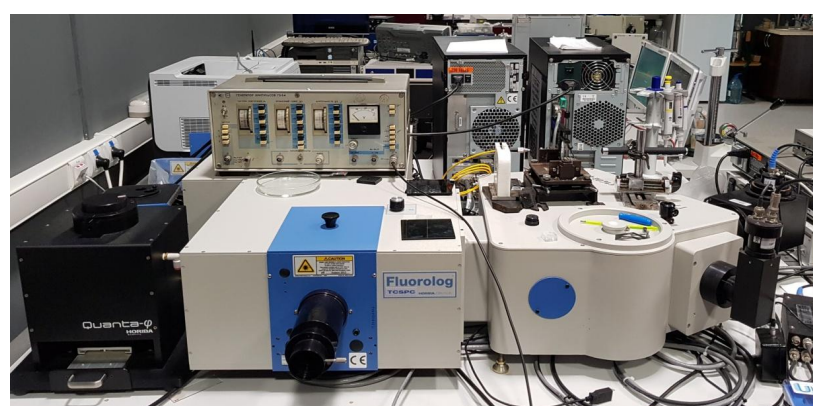

$\mathrm{b}$

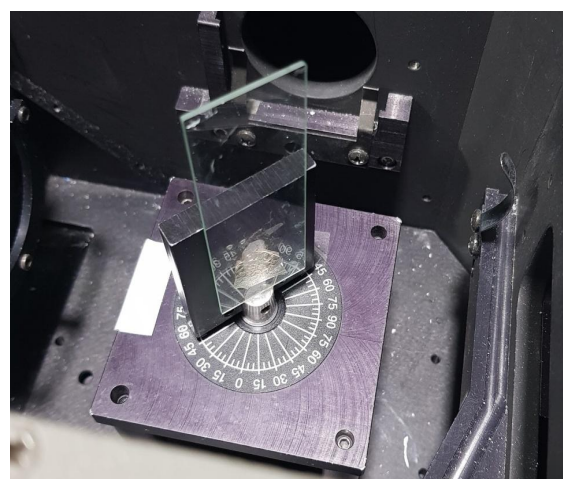

c

Fig. 2 (a) scheme of HORIBA Fluorolog-3 TCSPC; (b) modular system HORIBA Fluorolog-3 TCSPC; (c) sample of film in modular system HORIBA.

Spectral characteristics of PAH in aqueous solution and sorbed onto the solid surface were studied on the example of pyrene having similar luminescence spectra with BP. Pyrene was dissolved in a dimethyl sulfoxide (DMSO) solution at a concentration of $10^{-6} \mathrm{~mol} / \mathrm{l}$. Test sample solutions were prepared on distilled water 
$\mathrm{pH}=7$. The obtained films were kept in the prepared solution, then the samples were dried.

\section{Results and discussion}

The results of fluorescent analysis of films after static sorption in DMSO and pyrene solution are shown in Fig. 3. The resulting spectra are represented by five vibron bands in the wavelength range of $372 \mathrm{~nm}$ to $393 \mathrm{~nm}$, which represent the characteristic fluorescence spectrum of pyrene [19].

Films of pure chitosan are very brittle and not very convenient to use. Various additives are used to improve their mechanical properties [20].

In this work, modified matrices were developed using CTAB $(\mathrm{C}=0.01 \mathrm{M})$, and a solution of PVA (synthetic water-soluble polymer, $\mathrm{C}=5 \%$ ) was added to increase strength. Graphs show chitosan films are capable of effectively adsorbing pyrene, also the fluorescence intensity of modified films is significantly higher than that of unmodified chitosan film without additives.

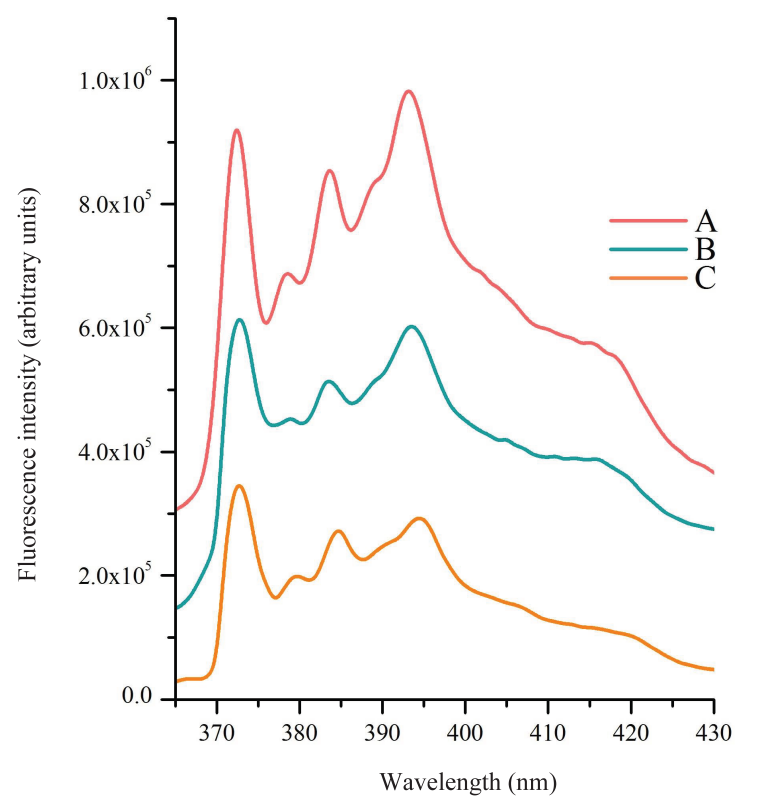

Fig. 3 Film fluorescence spectra: A - chitosan film modified with PVA and CTAB; B - chitosan film modified with PVA; $\mathrm{C}$ - chitosan film.

The system of vibrational levels of the pyrene molecule is sensitive to the properties of surrounding substances. In this regard, it is possible to use the method of fluorescent probes. Its essence is that luminescent molecules react to changes in the parameters of their microenvironment by changing the intensity of luminescence. This allows us to characterize the microenvironment around the probe molecules and evaluate their parameters that affect the luminescence of the probe [21].

In this case, the pyrene is a luminescent probe that shows the polarity of the microenvironment of molecules by means of ratio to the intensities of the first and third maxima of the fluorescence spectrum $\left(I_{1} / I_{3}\right)$. This value can be used to estimate the sorption ability of modified chitosan films and the effect of modifying substances on the intensity of pyrene fluorescence [19].

When the pyrene is sorbed from the DMSO solution to chitosan films, the polarity index decreases from 1.81 for the solution (Fig. 4, curve 0), up to 1.32 for chitosan film (Fig. 3, curve C) and up to 1.08 for chitosan film with the addition of CTAB (Fig. 3, curve A). The results are shown in Table 1. This indicates that the modified matrices are more hydrophobic than the unmodified matrices and they represent a good basis for the luminescent signal.

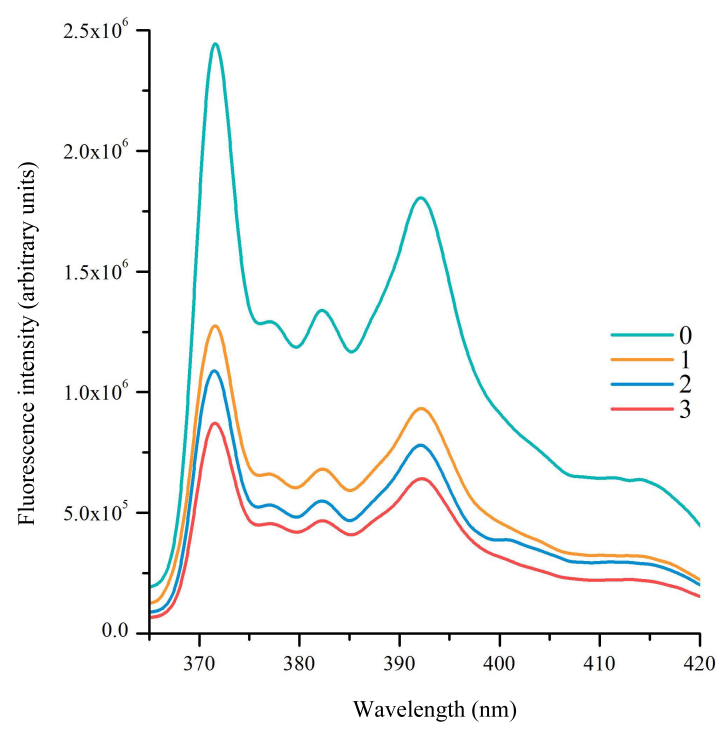

Fig. 4 Fluorescence spectra of solutions: 0 - initial solution; 1 - after static sorption $(\mathrm{t}=30 \mathrm{~min})$ with chitosan film; 2 - after static sorption $(t=30 \mathrm{~min})$ with chitosan-PVA film; 3 - after static sorption $(t=30 \mathrm{~min})$ with chitosan-PVA-CTAB film.

Table 1 Indexes of polarity.

\begin{tabular}{ccc}
\hline & Films type & $\begin{array}{c}\text { Index of } \\
\text { polarity, units }\end{array}$ \\
\hline A & Chitosan, PVA, CTAB & $1.08 \pm 0.03$ \\
\hline B & Chitosan, PVA & $1.21 \pm 0.04$ \\
\hline C & Chitosan & $1.32 \pm 0.04$ \\
\hline 0 & DMSO-solution & $1.81 \pm 0.05$ \\
\hline
\end{tabular}

Reliability and reproducibility of results of pyrene determination by SPL are confirmed by the calculations of extent of pyrene extraction at static sorption from initial solution. Fluorescence spectra of the initial and final samples of DMSO and pyrene solutions (before 
and after pyrene sorption) were measured to calculate the extent of pyrene extraction by the films. The results of solution analysis are shown in Fig. 4. The most effective matrix was modified PVA and CTAB and its extent of extraction was $64 \%$.

In Ref. [2], films of chitosan, cellulose and cellulose diacetate were made. The degree of pyrene extraction by these matrices was respectively 4,24 , and $15 \%$. The pyrene polarity indexes were respectively $1.22 \pm 0.02$, $1.23 \pm 0.01$ and $1.51 \pm 0.02$. The analysis of pyrene fluorescence spectra on matrices after sorption from a water-ethanol solutions demonstrated that the intensity of pyrene fluorescence is low, and the sorption capacity of matrices from cellulose diacetate and chitosan from a water-ethanol solution of pyrene is low. Similar results were presented by the authors in Refs. [22-23], where the value of the polarity index varied from 1.72 to 1.32 for different types of matrices.

In this work, the modification of chitosan films was carried out with PVA and CTAB. Based on the results of fluorescent analysis of pyrene in an aqueous solution of DMSO, it can be concluded the sorption capacity of the films significantly increases. The pyrene recovery rate on modified solid matrices was $64 \%$, which is more than in the presented literature data [2, 22-23]. The pyrene polarity index decreased to $1.08 \pm 0.03$, compared to $1.81 \pm 0.05$ in the initial DMSO solution, indicating greater hydrophobicity of the modified matrices. Thus, the using of the proposed solid matrices for TFL is enough effective.

The extent of pyrene extraction was calculated by Eq. (1):

$$
R=\frac{I_{\text {sol }}-I_{\text {film }}}{I_{\text {sol }}} \times 100 \%
$$

where $I_{s o l}$ is maximum luminescence intensity of the initial solution; $I_{\text {film }}$ is maximum intensity of solution luminescence after static film sorption.

The results of the calculations are shown in Table 2.

Table 2 Extent of pyrene extraction by films.

\begin{tabular}{ccc}
\hline & $\begin{array}{c}\text { Solutions after film } \\
\text { sorption }\end{array}$ & $\begin{array}{c}\text { Extent of pyrene } \\
\text { extraction from } \\
\text { solution by films, \% }\end{array}$ \\
\hline 1 & Chitosan & $48 \pm 2$ \\
\hline 2 & Chitosan, PVA & $53 \pm 3$ \\
\hline 3 & Chitosan, PVA, CTAB & $64 \pm 4$ \\
\hline
\end{tabular}

For the most effective modification of the matrices, graphs (Fig. 5) of dependence of the pyrene fluorescence intensity on the amount of CTAB at three pyrene concentrations: $10^{-5}, 10^{-6}$ and $10^{-7}$ were plotted. From the obtained graphs, it can be seen that when pyrene is sorbed from the DMSO solution onto a matrix modified by CTAB and PVA, the intensity of pyrene fluorescence increases significantly with an increase in the amount of CTAB in the film composition. The maximum values of pyrene fluorescence on the modified film are observed at the mass fraction of CTAB $\omega=15 \times 10^{-3} \%$. With further addition of CTAB, the pyrene fluorescence signal does not increase. Thus, it is advisable to use this amount of CTAB for effective analysis of pyrene using solid chitosan matrices.

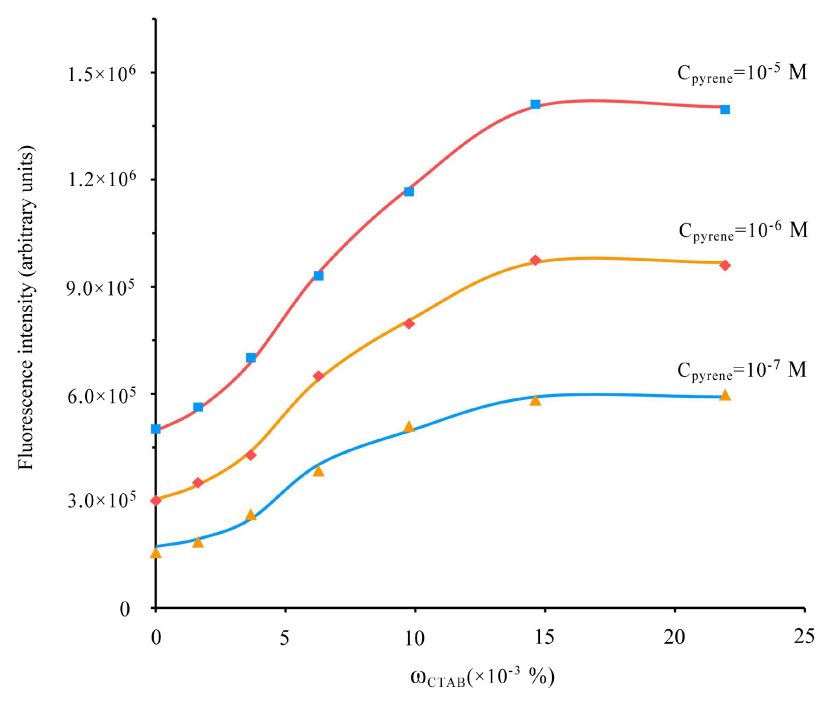

Fig. 5 Dependence of fluorescence intensity pyrene on chitosan-PVA-CTAB films on mass fraction CTAB.

These results can be explained by the fact that the polymer structure changes when PVA and CTAB are added to the chitosan solution. When biopolymers (chitosan) are mixed with synthetic polymers (PVA), new composite materials with new characteristics are formed. The modified chitosan film with PVA combines the properties of both polymers because hydrogen bonds are formed between the $\mathrm{OH}-$ and $\mathrm{NH}-$ chitosan groups and the $\mathrm{OH}$ - group at PVA. [24]

It is also known from the literature that chitosan is quite effective in forming complexes with surfactants. Chitosan is cationic biopolymer, and at addition of molecules to it anion surfactant, it forms strong communications due to Coulomb interaction [25].

At the same time, it loses charge, and the formed complex ceases to be soluble in water and precipitates. In our research when anionic surfactant was added, strong enough chitosan films failed to synthesize.

The addition of a cationic surfactant is also produced complexes that strongly alter the initial structure of the polymer chain and the physical properties of the polymer. The type of change depends on the ratio of polymer to surfactant concentrations. Complexes with whole micelles can be formed at micelle-forming surfactant concentrations higher than critical micelle formation concentration [26].

It can be assumed that CTAB molecules and micelles attach to the chitosan polymer chain and the matrix becomes more porous with more hydrophobic 
bonds, whereby pyrene attachment occurs more efficiently.

\section{Conclusion}

The results confirm the high solubilizing ability of solid matrices based on chitosan and allow using the hydrophobic properties of chitosan films in hydrophobic form for pyrene analysis. It was found that SPL analysis allows increasing the sensitivity of pyrene detection in aqueous media without additional extraction of substances and concentration with organic solvents. Also, when using solid matrices, it is possible to perform a luminescent analysis of substances with a large range of optical transparency.

The study of the spectral characteristics of the pyrene luminescent probe suggests that in films with CTAB, hydrophobic pyrene molecules are in a solubilized state inside micelles. Studying the parameters of pyrene adsorption from aqueous DMSO solutions allows us to conclude that pyrene molecules are sorbed on a solid matrix inside CTAB associates. Modification of chitosan matrices using PVA and CTAB allows us to increase the efficiency of concentration of pyrene molecules. Therefore, this allows improving the analytical characteristics of the luminescent pyrene detection method.

Further studies suggest using the results obtained for the quantitative determination of pyrene with the use of SPL with modified chitosan matrices in aqueous environment and food items. Also the results can be used to develop a method for determining other types of PAHs, such as BP, for using in test systems for environmental monitoring.

\section{Disclosures}

All authors declare that there is no conflict of interests in this paper.

\section{References}

1. H. I. Abdel-Shafy, M. S. M. Mansour, “A review on polycyclic aromatic hydrocarbons: Source, environmental impact, effect on human health and remediation", Egyptian Journal of Petroleum 25(1), 107-123 (2016).

2. A. V. Strashko, Razrabotka polisakharidnykh matrits i usloviy tverdofaznogo lyuminestsentnogo opredeleniya politsiklicheskikh aromaticheskikh uglevodorodov dlya ekologicheskogo monitoringa vodnykh sred, Dissertation, Saratov State University, 124 p. (2015) [in Russian].

3. K.-H. Kim, S. A. Jahan, E. Kabir, and R. J. C. Brown, "A review of airborne polycyclic aromatic hydrocarbons (PAHs) and their human health effects", Environment International 60, 71-80 (2013).

4. A. Ramesh, A. Archibong, D. Hood, Z. Guo, and B. Loganathan, "Global Environmental Distribution and Human Health Effects of Polycyclic Aromatic Hydrocarbons" Chapter in Global Contamination Trends of Persistent Organic Chemicals, 97-126 (2011).

5. "Maximum permissible concentrations of pollutants in the atmospheric air of urban and rural settlements", Resolution of the Government of the Russian Federation No. 165 on the approval of hygienic standards of the GN 2.1.6.3492-17, Amendment of 31.05.2018 [in Russian].

6. “About food safety", Technical Regulations of the Customs Union, 127-152 (2011) [in Russian].

7. S. R. Sokolnikova, D. A. Tatarinov, and N. A. Myslitskaya, "Change of luminescent properties of pyrene in surface-active substance solution using an albumin", Memoirs of the Faculty of Physics 4, 1941101 (2019) [in Russian].

8. S. M. Rogacheva, A. B. Shipovskaya, A. V. Strashko, T. I. Gubina, E. V. Volkova, and A. G. Melnikov, "Polysaccharide fibers as matrices for solid-surface fluorescence", International Journal of Polymer Science 2014, 183413 (2014).

9. D. A. Tatarinov, S. R. Sokolnikova, and N. A. Myslitskaya, "Influence of silver nanoparticles on the luminescent properties of pyrene in micellar solution", Electronic, spin and quantum processes in molecular and crystal systems: Theses of reports and presentations, BGPU, Ufa, 21 (2019) [in Russian].

10. T. Saitoh, H. Itoh, and M. Hiraide, "Admicelle-enhanced synchronous fluorescence spectrometry for the selective determination of polycyclic aromatic hydrocarbons in water", Talanta 79, 177-182 (2009).

11. W. B. Wilson, A. A. Costa, H. Wang, A. D. Campiglia, J. A. Dias, and S. C. L. Dias, "Preconcentration of water samples with BEA zeolite for direct determination of polycyclic aromatic hydrocarbons with laser-excited timeresolved Shpol'skii spectroscopy", Microchemical Journal 110, 246-255 (2013).

12. A. H. Ackerman, R. J. Hurtubise, "Methods for coating filter paper for solid-phase microextraction with luminescence detection and characterization of the coated filter paper by infrared spectrometry", Analytical Chemical Acta 474(1-2), 77-89 (2002).

13. A. I. Suvorova, A. L. Suvovrov, M. V. Ivanenko, and E. I. Shishkin, "Nanocomposite membrane films based on cellulose ethers and tetraethoxysilane", Russian Nanotechnologies 4(1-2), 154-161 (2009) [in Russian].

14. M. Rinaudo, "Chitin and chitosan: properties and applications", Progress in Polymer Science 31(7), 603-632 (2006). 
15. V. F. Adulin, "Technology and properties of chitosan from the river cancer shell", Journal of SGTU 4(16), 18-24 (2006) [in Russian].

16. M. Jabli, H. V. Baouab, M. S. Roudesli, and A. Bartegi, "Adsorption of acid dyes from aqueous solution on a chitosan-cotton composite material prepared by a new pad-dry process", Journal of Engineered Fibers and Fabrics 6(3), 1-12 (2011).

17. HORIBA Instruments Incorporated. Fluorolog-3 Operation Manual rev. (2014).

18. L. A. Currie, G. Svehla, "Nomenclature for the presentation of results of chemical analysis. (IUPAC Recommendations 1994)", Pure and Applied Chemistry 66(3), 595-608 (1994).

19. G. V. Mel'nikov, T. I. Gubina, and O. A. Dyachuk, "Influence of the polarity of the microenvironment of pyrene on the intensity of its solid-phase luminescence", Russian Journal of Physical Chemistry 80(7), 1160-1163 (2006).

20. S. N. Shtykov, "Chemical analysis in nanoreactors: Main concepts and applications", Journal of Analytical Chemistry 57(10), 859-868 (2002).

21. E. V. Naumova, A. G. Melnikov, and G. V. Melnikov, "Luminescence quenching by heavy metal ions of probes based on anthracene, pyrene, and eosin in human serum albumin", Journal of Applied Spectroscopy 80(2), 159-163 (2013).

22. L. Chen, D. Chen, and C. Wu, "A New Approach for the Flocculation Mechanism of Chitosan", Journal of Polymers and the Environment 11(3), 87-92 (2003).

23. L. Chen, D. Chen, and C. Wu, "Studying Flocculation Mechanism of Chitosan with Pyrene-fluorescence Probe Method", Chinese Journal of Chemistry 21, 1224-1228 (2003).

24. K. Choo, Y. C. Ching, C. H. Chuah, S. Julai, and N.-S. Liou, "Preparation and characterization of polyvinyl alcohol-chitosan composite films reinforced with cellulose nanofiber", Materials 9(8), 644 (2016).

25. M. Thongngam, D. J. McClements, "Characterization of Interactions between chitosan and an anionic surfactant", Journal of Agricultural and Food Chemistry 52(4), 987-991 (2004).

26. K. N. Bakeev, E. A. Ponomarenko, T. V. Shishkanova, D. A. Tirrell, A. B. Zezin, and V. A. Kabanov, "Complexation of amphiphilic polyelectrolytes with surfactants of the same charge in water solutions", Macromolecules 28(8), 2886-2892 (1995). 